\title{
Quantitative Diffusion and Spectroscopic Neuroimaging Combined with a Novel Early-Developmental Assessment Improves Models for 1-Year Developmental Outcomes
}

\author{
(D) H.G. Moss, DL.G. Wolf, P. Coker-Bolt, V. Ramakrishnan, (DT. Aljuhani, DM. Yazdani,
}

(D) T.R. Brown, (D).H. Jensen, and (DD.D. Jenkins

O-

\begin{abstract}
BACKGROUND AND PURPOSE: Preterm infants are at risk for overt and silent CNS injury, with developmental consequences that are difficult to predict. The novel Specific Test of Early Infant Motor Performance, administered in preterm infants at term age, is indicative of later developmental gross motor and cognitive scores at 12 months. Here, we assessed whether functional performance on this early assessment correlates with CNS integrity via MR spectroscopy or diffusional kurtosis imaging and whether these quantitative neuroimaging methods improve predictions for future 12-month developmental scores.
\end{abstract}

MATERIALS AND METHODS: MR spectroscopy and quantitative diffusion MR imaging data were acquired in preterm infants $(n=16)$ at term. Testing was performed at term and 3 months using the Specific Test of Early Infant Motor Performance and the Bayley Scales of Infant and Toddler Development, Third Edition, at 12 months. We modeled the relationship of MR spectroscopy and diffusion MR imaging data with both test scores via multiple linear regression.

RESULTS: MR spectroscopy NAA ratios at a TE of $270 \mathrm{~ms}$ in the frontal WM and basal ganglia and kurtosis metrics in major WM tracts correlated strongly with total Specific Test of Early Infant Motor Performance scores. The addition of MR spectroscopy and diffusion separately improved the functional predictions of 12-month outcomes.

CONCLUSIONS: Microstructural integrity of the major WM tracts and metabolism in the basal ganglia and frontal WM strongly correlate with early developmental performance, suggesting that the Specific Test of Early Infant Motor Performance reflects CNS integrity after preterm birth. This study demonstrates that combining quantitative neuroimaging and early functional movement improves the prediction of 12-month outcomes in premature infants.

ABBREVIATIONS: AIC = Akaike Information Criterion; adj- $R^{2}=$ Adjusted R-squared; Bayley-III = Bayley Scales of Infant and Toddler Development, Third Edition; $B G$ = basal ganglia; DKI = diffusional kurtosis imaging; $F A=$ fractional anisotropy; $g C C=$ genu of the corpus callosum; IFOF = inferior fronto-occipital fasciculus; KFA = kurtosis fractional anisotropy; PLIC = posterior limb of the internal capsule; PTR = posterior thalamic/optic radiations; $5 C C=$ splenium of the corpus callosum; STEP = Specific Test of Early Infant Motor Performance

$\mathbf{P}$ remature birth results in CNS dysmaturation characterized by altered microstructural WM and myelination not quantifiable on head sonography or qualitative MR imaging. ${ }^{1-3}$ Interventions for developmental delays are typically not started until later in infancy on failure to sit or walk, squandering a period of high neuroplasticity. We developed the Specific Test of Early Infant Motor Performance (STEP) to address this need and have shown that

Received June 17, 2021; accepted after revision September 27.

From the Department of Neuroscience (H.G.M., J.H.J.), Center for Biomedical Imaging (H.G.M., T.R.B., J.H.J., D.D.J.), Department of Pediatrics (L.G.W., D.D.J.), Division of Occupational Therapy (P.C.-B., T.A.), College of Health Sciences, Division of Public Health Sciences (V.R., T.A.), and Department of Radiology and Radiological Science (M.Y., T.R.B., J.H.J.), Medical University of South Carolina, Charleston, South Carolina.

This research was supported by the South Carolina Clinical \& Translational Research Institute at the Medical University of South Carolina, Clinical and Translational Science Awards National Institutes of Health/National Center for Advancing Translational Sciences ULITR000062 (to P. Coker-Bolt), and National Institutes of Health/National Institute of Neurological Disorders and Stroke F31NS108623 (to H.G. Moss)
STEP scores at term and 3 months can predict scores on the Bayley Scales of Infant and Toddler Development, Third Edition (Bayley-III) at 12 months. $^{4-6}$

A recent consensus statement emphasized combining neuroimaging with clinical assessment for improved diagnosis of cerebral palsy. ${ }^{7}$ In this study, we determined whether neuroimaging (MR spectroscopy or diffusion MR imaging) reflects STEP performance at term and improves the ability of STEP to predict later development. MR spectroscopy quantifies mobile intracellular metabolomics in the basal ganglia (BG) and frontal WM regions

Please address correspondence to Hunter Moss, BS, Center for Biomedical Imaging, Department of Neuroscience, Medical University of South Carolina, 173 Ashley Ave, Room 403, Charleston, SC 29425; e-mail: mossh@musc.edu

- Indicates open access to non-subscribers at www.ajnr.org

Indicates article with online supplemental data.

http://dx.doi.org/10.3174/ajnr.A7370 

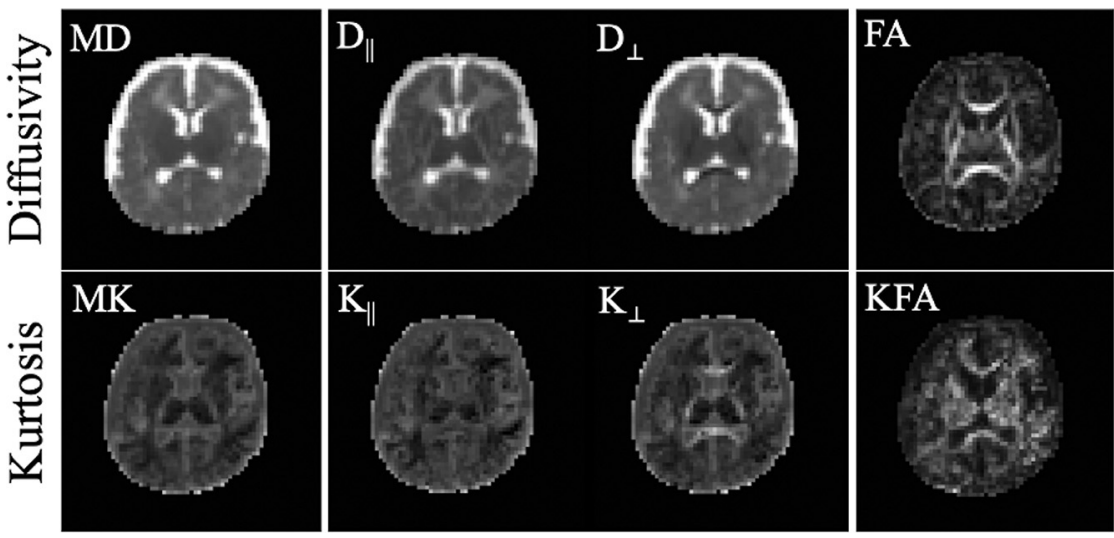

FIG 1. An axial section from a representative subject showing rotationally invariant tensor quantities calculated from the DKI analysis. The upper row shows the mean, axial, and radial diffusivities and fractional anisotropy (MD, $D_{\|}, D_{\perp}$, and FA, respectively). The lower row shows the corresponding kurtosis metrics (MK, $\mathrm{K}_{\|}, \mathrm{K}_{\perp}$, and $\mathrm{KFA}$ ). Diffusivity quantifies the degree of water mobility, while kurtosis is a measure of the complexity of water diffusion within the tissue microenvironment. Kurtosis provides more detail in the sparsely myelinated infant brain. The $M D, D_{\|}$, and $D_{\perp}$ have units of square micrometer/millisecond with the remaining metrics being unitless. MD indicates mean diffusivity; $D_{\|}$, axial diffusivity; $\mathrm{D}_{\perp}$, radial diffusivity; $\mathrm{MK}$, mean kurtosis; $\mathrm{K}_{\|}$, axial kurtosis; $\mathrm{K}_{\perp}$, radial kurtosis.

Image Acquisition and Processing We performed MRIs at 38-44 weeks on a $3 \mathrm{~T}$ Tim Trio scanner (Siemens) on unsedated infants, as previously described. ${ }^{24}$ We acquired single-voxel ${ }^{1} \mathrm{H}-\mathrm{MR}$ spectra using point-resolved spectroscopy $(\mathrm{TR}=2000 \mathrm{~ms}, \mathrm{TE}=30$ and $270 \mathrm{~ms}, 128$ signal averages). Data were acquired using an isotropic voxel $(15 \mathrm{~mm})^{3}$ in the left BG and right frontal WM (Online Supplemental Data) for $\mathrm{TE}=30 \mathrm{~ms}$ and in the right frontal $\mathrm{WM}$ for $\mathrm{TE}=270 \mathrm{~ms}$. A single voxel $(14.37 \mathrm{~mm})^{3}$ was extracted from the left BG region of a multivoxel point-resolved spectroscopy chemical shift imaging scan $(\mathrm{TR}=1700 \mathrm{~ms}$, $\mathrm{TE}=270 \mathrm{~ms}, 16 \times 16$ grid, and 1 signal average). All voxels were placed (or extracted) in regions that had no apparent lesions.

The LCModel ${ }^{25}$ (http://www. lcmodel.com/) quantified metabolite

affected by neonatal injury that relate to outcome. ${ }^{8-12}$ Both diffusional kurtosis imaging (DKI) ${ }^{13}$ and $\mathrm{DTI}^{14}$ are diffusion MR imaging methods that quantify tissue integrity through the random movement of water molecules. Kurtosis quantifies the water movement deviation away from and not represented by the strict Gaussian distribution that DTI imposes. ${ }^{13}$ DKI measures tissue heterogeneity (intra- or extracellular barriers) ${ }^{15}$ and is acutely sensitive to pathologic processes. ${ }^{16}$ While application of DKI to brain development is new, ${ }^{17-21}$ studies in typically developing children show nonlinear relationships of kurtosis with age. ${ }^{19-21}$ The kurtosis tensor provides higher-order CNS structural information on the organization of the microenvironment, which may directly impact development.

We hypothesized that lower metabolic or microstructural integrity identifies dysmaturation that manifests as functional developmental performance at term or 3 months by the STEP and at 12 months by the Bayley-III. ${ }^{4,12}$ This investigation provides evidence that quantitative neuroimaging paired with early developmental assessment improves the prediction of later-stage development.

\section{MATERIALS AND METHODS \\ Sample}

We enrolled infants $(n=16)$ born at 24-34 weeks gestational age with institutional review board approval and parental informed consent, in accordance with the Declaration of Helsinki.

\section{Developmental Assessments}

The STEP was administered at term (37-42 weeks) and at 3 months. The STEP is composed of 10 items with a maximum total score of 30. STEP cutoff scores for low and below normal at 12 months and Bayley-III scores are $\leq 16$ (term) and $\leq 22$ ( 3 months). ${ }^{6}$

We assessed neurodevelopment at 12 months by the Bayley-III $(n=15)$. Below normal or low Bayley-III gross motor and cognitive scaled scores are defined as $<9$ and 1 SD below the mean. ${ }^{22,23}$ ratios to creatine using a simulated metabolite basis set $^{26}$ generated with the VeSPA software package ${ }^{27}$ (https:/github.com/vespa-mrs/ vespa) with any additional metabolite ratios computed from these (Online Supplemental Data). Processed spectra were visually inspected for quality of fit, and the metabolite ratio inclusion criterion was the Cramer-Rao lower bound of $<20 \%$. For the TE $=$ 30 ms spectra, the mean (SD) (median, minimum-maximum) of the full width at half maximum was 0.044 (0.033) (0.041, 0.014$0.114)$ with an SNR of 10 (3) $(10,4-14)$ for the BG and 0.055 (0.038) $(0.043,0.01-0.114)$ with an SNR of $10(3)(9,6-16)$ for the $\mathrm{WM}$. For the $\mathrm{TE}=270 \mathrm{~ms}$ spectra, the full width at half maximum was $0.069(0.039)(0.060,0.014-0.143)$ for the BG with an SNR of 8 (3) $(8,4-14)$ and $0.045(0.032)(0.038,0.010-0.124)$ for the WM with an SNR of 5 (2) (4, 2-9).

We obtained DKI data with $(2.7 \mathrm{~mm})^{3}$ isotropic voxels, with $\mathrm{TE}=99 \mathrm{~ms}, \mathrm{TR}=4800 \mathrm{~ms}$, and $b=0,1000$, and $2000 \mathrm{~s} / \mathrm{mm}^{2}$ with 64 diffusion-encoding directions per nonzero b-value shell, 37 contiguous axial slices per image volume, and 10 additional non-diffusion-weighted images $\left(b=0 \mathrm{~s} / \mathrm{mm}^{2}\right)$.

After removing image volumes and gradient directions corrupted by excessive head motion (on average, 13 [12] volumes were removed), we used the DESIGNER pipeline ${ }^{28-34}$ (https:// pydesigner.readthedocs.io/en/latest/) to perform a $\mathrm{DKI}^{35}$ analysis, which calculated fractional anisotropy (FA), mean, axial, and radial diffusivities; and mean, axial, and radial kurtosis and kurtosis FA (KFA). ${ }^{13,36-39}$ DKI data were fit globally to obtain the best estimates for both the diffusion and kurtosis tensors along with their associated diffusion metrics (Fig 1).

\section{ROI Placement}

Bilateral WM ROIs $(n=8)$ were manually drawn using each subject's FA image for anatomic reference in the anterior limb and posterior limb of the internal capsule (PLIC), external capsule, uncinate fasciculus, inferior fronto-occipital fasciculus (IFOF), 

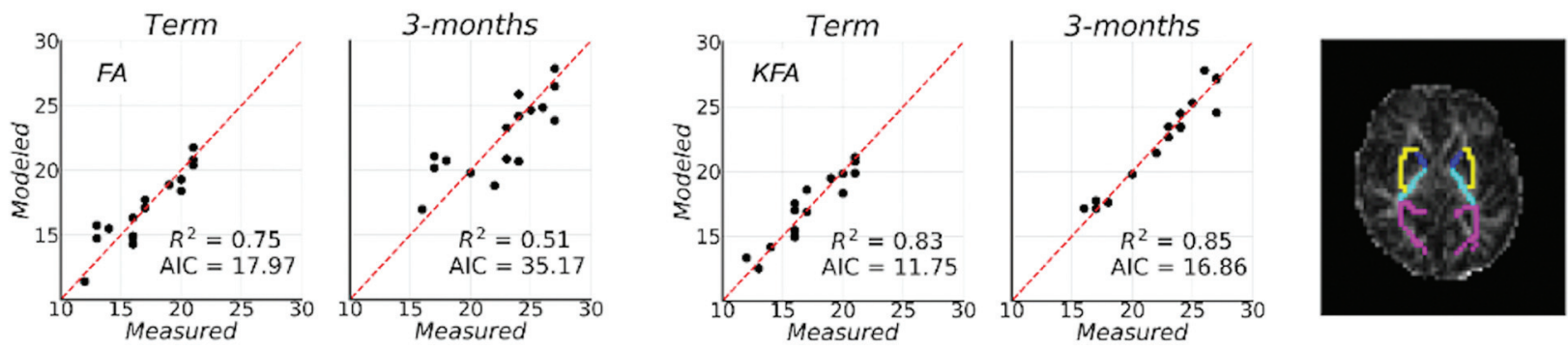

FIG 2. Plots showing measured STEP scores at term or 3 months versus modeled STEP scores using FA for diffusivity (left columns) or KFA for kurtosis (right columns) to predict STEP. KFA and FA models show the best fit to measured STEP scores primarily from combining metrics in the external capsule (yellow) and the PTR (purple). Goodness-of-fit metrics of adj- $R^{2}$ and AIC are noted with each model. While both FA and KFA predict STEP function during 3 months of development, KFA performs better in the model than FA (higher $R^{2}$ and lower AIC); for STEP at term: $\Delta$ AIC $=$ 6.22, $P<.05$; and for STEP at 3 months: $\triangle \mathrm{AIC}=18.31, P<.001$. All models are corrected for gestational age at birth and at MR imaging.

posterior thalamic/optic radiations (PTR), and the corpus callosum (genu/splenium [gCC]/[sCC], Online Supplemental Data). A clinical pediatric neuroradiologist verified correct ROI placement. Last, a total WM ROI was created by combining these individual ROIs.

\section{Statistical Analysis}

Multiple linear regression (SAS 3.8; SAS Institute) was performed with STEP at term or 3 months as the response variable and metabolite ratios from $\mathrm{TE}=30$ or $270 \mathrm{~ms}$ or DKI-derived metrics as predictor variables. Gestational age at birth and MR imaging were included in all models to account for degree of prematurity.

For the DKI metrics, STEP was modeled in two ways:

1. For a single ROI, each diffusion parameter was averaged separately over all voxels and included as an independent covariate $\left(\mathrm{eg}, \mathrm{STEP}_{\text {term }}=\mathrm{FA}_{\mathrm{sCC}}+\mathrm{KFA}_{\mathrm{sCC}}\right)$. This approach yields a parsimonious model in which a single WM tract could be sampled with several different diffusion parameters to model prognosis in a clinical setting.

2. To assess whether global brain dysmaturity or injury is more important to functional outcome prediction, we used a single diffusion metric averaged within each ROI as the covariates in the models (eg, $\left.\mathrm{STEP}_{\text {term }}=\mathrm{FA}_{\mathrm{sCC}}+\mathrm{FA}_{\mathrm{PTR}}+\mathrm{FA}_{\mathrm{PLIC}}\right)$. This allowed us to determine whether an individual diffusivity or kurtosis parameter in major WM tracts could improve model predictions for parsimonious sampling compatible with clinical settings.

\section{Model Selection}

We selected models for the best goodness of fit via larger adjusted R-squared $\left(\operatorname{adj}-R^{2}\right)$ and smaller Akaike Information Criterion (AIC). The difference in the AIC from full-versus-simpler models $\left(\Delta_{\text {AIC }}\right)$ was calculated with a $P$ value, $P=\exp \left(-\Delta_{\text {AIC }} / 2\right){ }^{40}$ The model that minimized the $\mathrm{AIC}^{41}$ and maximized the $\Delta_{\mathrm{AIC}}$ between full and simple models with $P<.05$ was selected as the most parsimonious model.

\section{RESULTS}

\section{Demographics}

MR spectroscopy ( $n=12$ or 16$)$ and DKI $(n=16)$ scans met our inclusion criteria. The mean age at MR imaging was 41.9
(1.5) weeks. Many infants had CNS conditions that may affect neurodevelopment (Online Supplemental Data).

\section{MR Spectroscopy $(\mathrm{NAA} / \mathrm{Cho})_{B G}$ and $(\mathrm{NAA} / \mathrm{Cr})_{W M}$ at TE = $\mathbf{2 7 0}$ ms Strongly Correlate with STEP Performance}

We assessed whether STEP performance represents concurrent MR spectroscopy metabolomics in the BG or frontal WM. After adjusting for gestational age at birth and MR imaging, (NAA/Cho) $)_{\mathrm{BG}}$ quantified at $\mathrm{TE}=270 \mathrm{~ms}(n=12)$ strongly correlated $(P=.04)$ with term STEP: $\beta=5.83$, adj- $R^{2}=0.82, \mathrm{AIC}=11.32, P_{\text {model }}<$ .001 ; and $(\mathrm{NAA} / \mathrm{Cr})_{\mathrm{WM}}$ correlated $(P=.04)$ with 3-month STEP: $\beta=9.76$, adj- $R^{2}=0.82, \mathrm{AIC}=16.11, P_{\text {model }}<.001$. A correlation was observed with the TE $=30 \mathrm{~ms}$ MR spectroscopy $(n=16)$ and STEP term $\left(\operatorname{adj}-R^{2}=0.77, \mathrm{AIC}=16.76, P_{\text {model }}=.02\right)$, but unlike the compact $\mathrm{TE}=270 \mathrm{~ms}$ models, it was composed of $5 \mathrm{WM}$ and 2 BG metabolite ratios (Online Supplemental Data).

\section{KFA and Inferior Fronto-Occipital Fasciculus Correlate Best with STEP Performance}

We found a strong linear relationship between diffusivity or kurtosis parameters and STEP scores (Online Supplemental Data). The Online Supplemental Data provide details of the $\beta$-value estimates for each WM ROI in the model fits. Of note are FA and KFA (Fig 2): KFA showed a notable improvement over FA in predicting concurrent functional performance, though FA models included fewer ROIs (Fig 2 and Online Supplemental Data). The KFA also outperformed all the other diffusion metrics at both STEP time points. Most metric models resulted in models with adj- $R^{2}>0.70$, indicating very strong correlations.

For the ROI-centered approach, we found that many individual WM tracts had moderately strong linear relationships with STEP scores (adj- $R^{2} \geq 0.60$, Online Supplemental Data). IFOF modeled the STEP term $\left(\operatorname{adj}-R^{2}=0.86, \mathrm{AIC}=8.86\right)$, and $\mathrm{gCC}$ modeled the STEP 3-month (adj- $\left.R^{2}=0.74, \mathrm{AIC}=25.38\right)$, similar to the total WM ROI (adj- $\left.R^{2}=0.74, \mathrm{AIC}=25.47\right)$ across diffusion metrics (Online Supplemental Data). Again, kurtosis metrics contributed more than diffusivity to the models (Online Supplemental Data). 

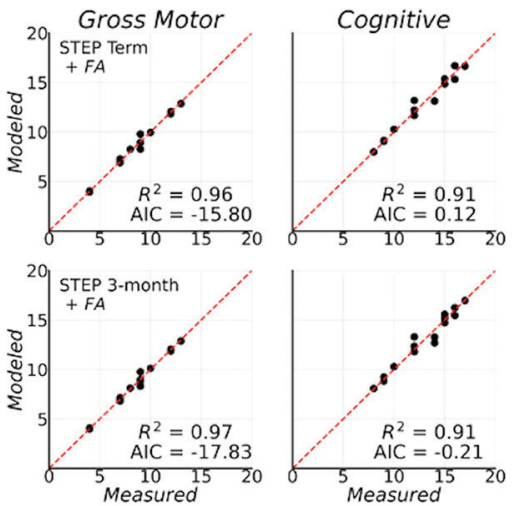
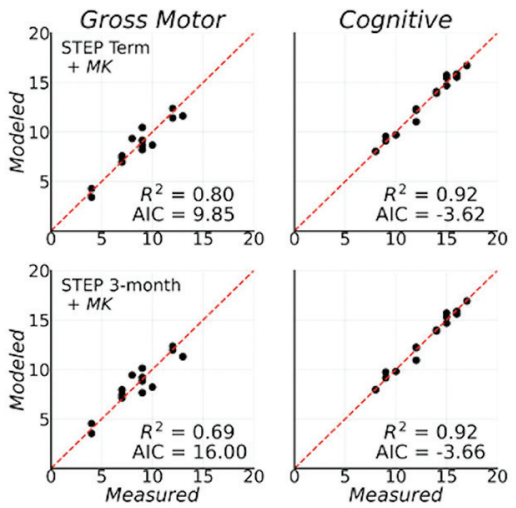
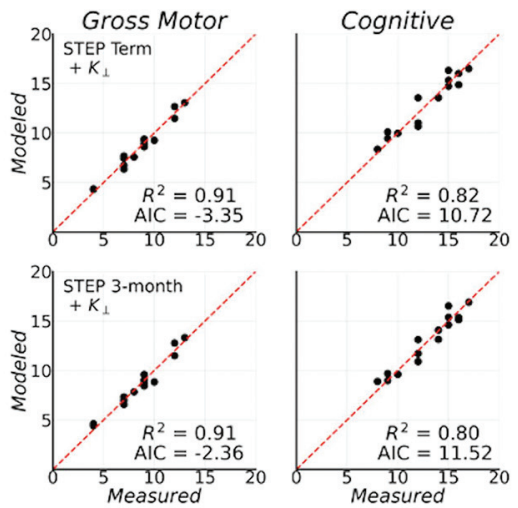

FIG 3. Measured 12-month Bayley-III gross motor (left columns) and cognitive scores (right columns) compared against the modeled Bayley scores, using an individual diffusivity (upper rows) or kurtosis (lower rows) parameter across various WM ROIs. By means of this diffusion parameter approach, FA (left), MK (center), and $\mathrm{K}_{\perp}$ (right) combined with STEP strongly predict both motor and cognitive Bayley scores, adjusted for gestational age at birth and MR imaging. Other metrics also predicted future function when combined with STEP scores (see the Online Supplemental Data for details on the optimal combination of WM ROls for each diffusion metric [ $\beta$-values, highest adj- $R^{2}$ and lowest AIC]). MK indicates mean kurtosis; $\mathrm{K}_{\perp}$, radial kurtosis.

\section{Both MR Spectroscopy and DKI Improve STEP Prediction of Bayley-III Scores}

Combining MR spectroscopy with STEP scores predicted BayleyIII motor and cognitive scores markedly better than STEP scores alone (Online Supplemental Data). For $\mathrm{TE}=270 \mathrm{~ms}$ data, improved motor score prediction models were found for term $\operatorname{STEP}\left(\Delta_{\mathrm{AI} C}=33.02, P<.001\right)$ and for 3-month STEP $\left(\Delta_{\mathrm{AIC}}=\right.$ 22.63, $P<.001)$. In modeling cognitive scores with $\mathrm{TE}=$ $270 \mathrm{~ms}$, (NAA/Cho) $)_{\mathrm{BG}}$ improved predictions beyond STEP alone (term: $\Delta_{\text {AIC }}=17.09 ; 3$ months: $\Delta_{\text {AIC }}=32.05$; both $P<.001$ ). MR spectroscopy at $\mathrm{TE}=30 \mathrm{~ms}$ improved STEP predictive models of cognitive scores (term: $\Delta_{\mathrm{AIC}}=15.36 ; 3$ months: $\Delta_{\mathrm{AIC}}=27$; both $P<.001)$ but included multiple metabolite ratios that detracted from a parsimonious model, unlike the $\mathrm{TE}=270 \mathrm{~ms}$ models, which included a single ratio. Model $\beta$-value estimates for each metabolite ratio included are detailed in the Online Supplemental Data.

We found that FA and radial and mean kurtosis were the best diffusion metrics to combine with STEP scores in predicting Bayley-III scores (Fig 3). For gross motor scores, FA improved the STEP-alone model at term and 3 months (both $\Delta_{\text {AIC }}>41, P<.001$ ) as did radial kurtosis (both $\Delta_{\text {AIC }}>26, P$ $<$.001). For cognitive scores, FA improved the STEP-alone model at term and 3 months (both $\Delta_{\mathrm{AIC}}>4, P<.001$ ), as did mean kurtosis (both $\Delta_{\text {AIC }}>27, P<.001$ ). Other DKI-derived metrics also predicted Bayley-III scores with STEP time points. (Online Supplemental Data). Included WM ROIs and $\beta$-value estimates for each are listed in the Online Supplemental Data.

For the ROI-centered approach, various combinations of kurtosis and diffusivity metrics within individual ROIs improved the predictions of Bayley-III gross motor and cognitive scores compared with the STEP-only models. For parsimony and simplicity, models using either PTR and IFOF with STEP scores had the lowest AICs for predicting Bayley Motor scores (Fig 4) and improved the model fit over the STEP alone (at term: $\Delta_{\text {AIC }}>30$; $P<.001$; at 3 months: $\left.\Delta_{\mathrm{AIC}}>28 ; P<.001\right)$. The gCC and external capsule with STEP scores improved prediction in Bayley cognitive scores (Online Supplemental Data) over STEP alone (at term: $\Delta_{\text {AIC }}>18 ; P<.001$; and at 3 months: $\Delta_{\text {AIC }}>14 ; P<$ $.001)$. Model fits and $\beta$-value estimates for each diffusion metric covariate included in the selected models for each WM ROI are provided in the Online Supplemental Data.

\section{DISCUSSION}

During early life after preterm birth, brain injuries as well as alterations in gray and WM maturation occur that influence later development of motor and cognitive skills. Influencing neuroplasticity to improve outcomes requires understanding the neuroimaging representations of brain injuries and their relation to functional movements during early infancy. Using MR spectroscopy and DKI parameters at term-age equivalent, we show that metabolomics for healthy neurons and microstructural integrity correspond to concurrent performance on the STEP early developmental assessment and that neuroimaging combined with the STEP improves prediction of future motor and cognitive scores compared with the STEP alone. Better predictive models would allow testing interventions at a very early period to optimally harness neuroplasticity. Our data highlight the potential for prognostication using the STEP and term-age quantitative neuroimaging and serve as a guide for metric selection. Our data provide proof of concept that quantitative MR imaging of the developing brain is vitally important in prognosis.

MR spectroscopy at TE $=270 \mathrm{~ms}$ in either the frontal WM or BG strongly related to STEP and NAA, and Cho ratios improved STEP predictive models for both gross motor and cognitive Bayley-III scores (Online Supplemental Data). However, we observed no relation or benefit with NAA or other metabolite ratios obtained from point-resolved spectroscopy sequence spectra at $\mathrm{TE}=30 \mathrm{~ms}$. This discrepancy may be due to a more reliable quantification of NAA and Cho at a long TE, in which there are fewer overlapping metabolite peaks, as has previously been reported. ${ }^{26}$ As markers of neuronal metabolic health ${ }^{42}$ and myelination, NAA and Cho are both complementary and additive in our models. ${ }^{1,9-11}$ Lower concentrations of NAA in the deep gray nuclei of the BG reflect metabolic impairment, lower neuronal 

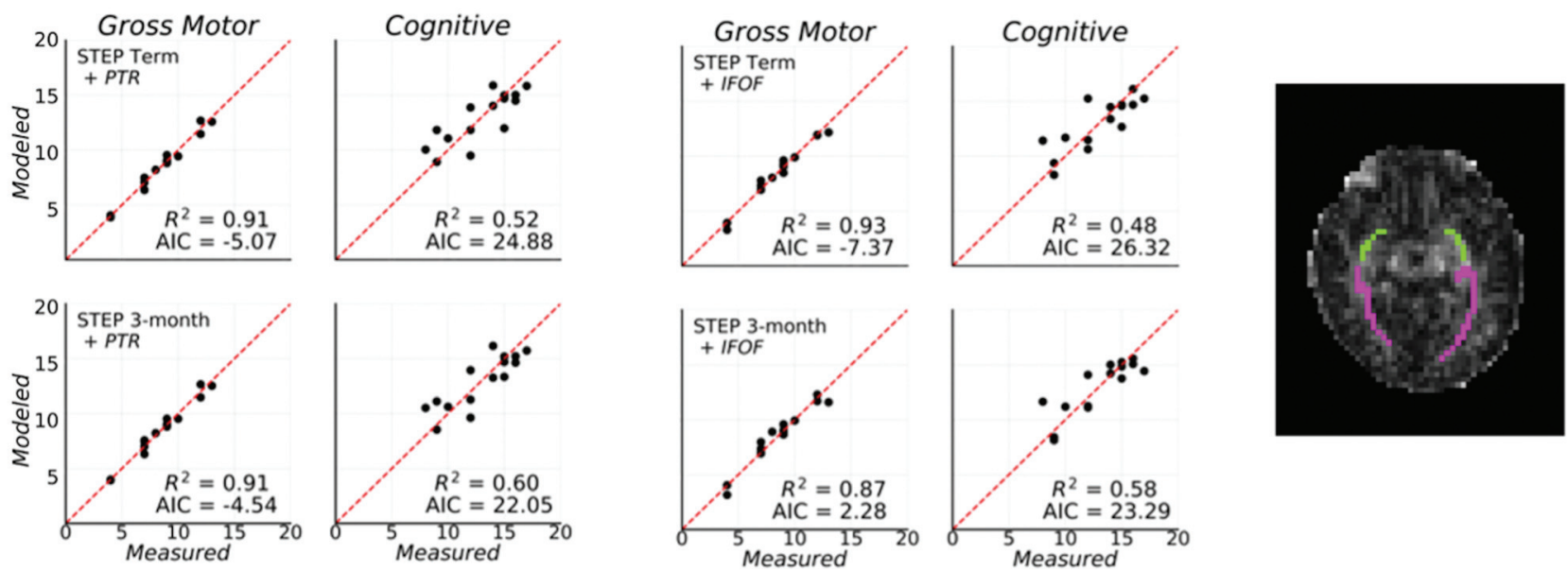

FIG 4. Measured 12-month Bayley-III gross motor (left columns) and cognitive scores (right columns) plotted against the modeled Bayley scores using STEP at term (upper rows) or 3 months (lower rows) + diffusivity and kurtosis metrics within individual WM ROIs to predict function. By means of this ROI-centered approach, the PTR and IFOF metrics combined with the STEP score result in the best predictive models, adjusted for gestational age at birth and MR imaging. Compared with STEP-alone predictive models (Online Supplemental Data), diffusivity and kurtosis in individual WM ROIs greatly improve the STEP model fit for both Bayley-III gross motor and cognitive test scores at 12 months. $\beta$-value estimates are provided in the Online Supplemental Data.

volume or density, and possibly insufficient dendritic arborization in the thalamus, responsible for sensory-input integration and regulation of voluntary movement. ${ }^{43}$ In the WM, lower NAA is associated with decreased axonal integrity in neonatal WM disease $^{10,44}$ and worse developmental outcomes in both preterm and term infants. ${ }^{9,11,12}$

ROIs (IFOF, PTR, and gCC) were also strongly correlated with STEP performance (Online Supplemental Data), indicating that visuomotor and sensorimotor integration pathways are critical in the early development of motor skills. IFOF and PTR with the STEP also predicted later motor function, while the gCC and the external capsule contributed strongly to STEP prediction for later cognitive function (Fig 4 and Online Supplemental Data). The best WM tracts for prognostication from our study (IFOF, PTR, and gCC) are readily identified, and ROIs are easily placed by clinical neuroradiologists on standard anatomic MR images or ROIs registered with an appropriate WM atlas.

Most quantitative clinical investigations use diffusion tensorderived FA that measures the directional dependence of water diffusion in a Gaussian distribution. FA increases nonlinearly with axon myelination, packing, and maturation that plateaus around 2 years of age. ${ }^{19,21}$ However, the diffusion tensor neither accounts for non-Gaussian cellular factors nor fully characterizes microstructure across the spectrum of development. In estimating the kurtosis tensor, we captured the non-Gaussian spin displacement that corresponds to cellular and tissue heterogeneity within the WM microstructure, different from DTI. ${ }^{13,16,45}$ Kurtosis metrics show a nonlinear relationship with age ${ }^{19,21}$ and increase with intracellular complexity, cell density, and myelination due to further restrictions on water movement. Radial kurtosis, in particular, increases more quickly during development than its diffusivity counterpart because myelin restricts diffusion across the axon. ${ }^{21}$ KFA is mathematically analogous to FA, but the kurtosis tensor provides complementary, more detailed directional variation on anisotropy information. ${ }^{37,38}$ Thus, unlike FA, KFA measurements are sensitive to higher-order angular variations in WM fiber orientations, ${ }^{36,38}$ also found in fiber-crossing regions. ${ }^{37}$

In our study, KFA outperformed FA in representing early function via STEP performance (Figs 2 and Online Supplemental Data), suggesting that complex WM cellular barriers or fibercrossings are important in the early ability to move with normal tone. Although not widely used, kurtosis metrics may be better markers of WM tract injury and health in the largely unmyelinated brain of infants than diffusivity metrics such as FA. When predicting future gross motor and cognitive performance (Online Supplemental Data), however, FA provided better results, followed closely by the KFA, radial kurtosis (gross motor), and mean kurtosis (cognitive). Taken together, our data support kurtosis information possibly significantly adding to conventional FA in understanding the complexity of WM development at term age.

As a standard protocol on clinical MR imaging machines, MR spectroscopy can yield high-quality results in neonatal studies due to high water content of brain tissue and low iron deposition. MR spectroscopy can be challenging in a clinical environment in unsedated neonates. Therefore, clinicians and MR imaging technicians should attempt to schedule MR spectroscopy scans after a feeding to encourage sleep and should be cognizant of when to repeat scans (ie, motion artifacts) or modify the imaging setup (eg, better swaddling/head immobilization). Furthermore, on the basis of our findings, long-TE spectral quantification is robust (ie, a more stable baseline and less confounding peaks) in quantifying a large concentration of metabolites (namely, NAA, Cho, and Cr). Conversely, shorter-TE MR spectroscopy will have a better SNR and more information on a small concentration of metabolites compared with long-TE sequences, with a trade-off in difficulties in quantification of overlapping peak shapes.

For more advanced diffusion imaging, DKI is easily performed within $4-6$-minutes by adding the b-value $2000 \mathrm{~s} / \mathrm{mm}^{2}$ to standard DTI protocols with 30 directions per nonzero b-value. Numer- 
ous open-source platforms (eg, Diffusional Kurtosis Estimator, ${ }^{33}$ https://github.com/m-ama/DKE, DESIGNER, ${ }^{28} /$ pyDesigner, and https://pydesigner.readthedocs.io/en/latest/) are available that provide semiautomated off-line DKI analysis.

Limitations of our study include our sample size, which precluded assessing whether brain dysmaturity or specific sites of injury were more important in neuroimaging models and any contributions of sex. Also, 12 months is a typical time for general developmental delays to manifest but is not optimal for diagnosing cerebral palsy. Another limitation is the combination of single-voxel and CSI data for the TE $=270 \mathrm{~ms}$ MR spectroscopy data modeling, which had slightly different TR values as well as differing spectral and shim quality. Last, MR spectroscopy voxel placement and WM ROI voxels were selected to reflect "apparently healthy" WM, but it is possible that lesions (Online Supplemental Data) may not have been readily visible during data gathering and analysis.

\section{CONCLUSIONS}

Our neuroimaging data provide proof of concept that the STEP reflects preterm brain dysfunction, dysmaturation, and/or injury. These data suggest that either MR spectroscopy or DKI can augment the STEP at term age in the prediction of long-term motor and cognitive development. If validated in a larger cohort, quantitative neuroimaging and STEP assessments may be used as surrogate end points in clinical trials and may facilitate earlier intervention for infants at high risk of developmental delays.

Disclosure forms provided by the authors are available with the full text and PDF of this article at www.ajnr.org.

\section{REFERENCES}

1. Lean RE, Han RH, Smyser TA, et al. Altered neonatal white and gray matter microstructure is associated with neurodevelopmental impairments in very preterm infants with high-grade brain injury. Pediatr Res 2019;86:365-74 CrossRef Medline

2. Back SA, Miller SP. Brain injury in premature neonates: a primary cerebral dysmaturation disorder? Ann Neurol 2014;75:469-86 CrossRef Medline

3. Duerden EG, Foong J, Chau V, et al. Tract-based spatial statistics in preterm-born neonates predicts cognitive and motor outcomes at 18 months. AJNR Am J Neuroradiol 2015;36:1565-71 CrossRef Medline

4. Coker-Bolt P, Barbour A, Moss $\mathrm{H}$, et al. Correlating early motor skills to white matter abnormalities in preterm infants using diffusion tensor imaging. J Pediatr Rehabil Med 2016;9:185-93 CrossRef Medline

5. Coker-Bolt P, Woodbury ML, Perkel J, et al. Identifying premature infants at high and low risk for motor delays using motor performance testing and MRS. J Pediatr Rehabil Med 2014;7:219-32 CrossRef Medline

6. Gower L, Jenkins D, Fraser JL, et al. Early developmental assessment with a short screening test, the STEP, predicts one-year outcomes. J Perinatol 2019;39:184-92 CrossRef Medline

7. Novak I, Morgan C, Adde L, et al. Early, accurate diagnosis and early intervention in cerebral palsy: advances in diagnosis and treatment. JAMA Pediatr 2017;171:897-907 CrossRef Medline

8. Chau V, Synnes A, Grunau RE, et al. Abnormal brain maturation in preterm neonates associated with adverse developmental outcomes. Neurology 2013;81:2082-89 CrossRef Medline

9. Kreis R, Hofmann L, Kuhlmann B, et al. Brain metabolite composition during early human brain development as measured by quantitative in vivo $\mathbf{1} \mathbf{H}$ magnetic resonance spectroscopy. Magn Reson Med 2002;48:949-58 CrossRef Medline

10. Tanifuji S, Akasaka M, Kamei A, et al. Temporal brain metabolite changes in preterm infants with normal development. Brain Dev 2017;39:196-202 CrossRef Medline

11. Kendall GS, Melbourne A, Johnson S, et al. White matter NAA/Cho and $\mathrm{Cho} / \mathrm{Cr}$ ratios at MR spectroscopy are predictive of motor outcome in preterm infants. Radiology 2014;271:230-38 CrossRef Medline

12. Lally PJ, Montaldo P, Oliveira V, et al. MARBLE consortium magnetic resonance spectroscopy assessment of brain injury after moderate hypothermia in neonatal encephalopathy: a prospective multicentre cohort study. Lancet Neurol 2019;18:35-45 CrossRef Medline

13. Jensen JH, Helpern JA. MRI quantification of non-Gaussian water diffusion by kurtosis analysis. NMR Biomed 2010;23:698-710 CrossRef Medline

14. Basser PJ, Mattiello J, LeBihan D. MR diffusion tensor spectroscopy and imaging. Biophys J 1994;66:259-67 CrossRef Medline

15. Jensen JH, Helpern JA, Ramani A, et al. Diffusional kurtosis imaging: the quantification of non-Gaussian water diffusion by means of magnetic resonance imaging. Magn Reson Med 2005;53:1432-40 CrossRef Medline

16. Steven AJ, Zhuo J, Melhem ER. Diffusion kurtosis imaging: an emerging technique for evaluating the microstructural environment of the brain. AJR Am J Roentgenol 2014;202:W26-33 CrossRef Medline

17. Galdi $\mathrm{P}$, Blesa M, Stoye DQ, et al. Neonatal morphometric similarity mapping for predicting brain age and characterizing neuroanatomic variation associated with preterm birth. Neuroimage Clin 2020;25:102195 CrossRef Medline

18. Gao J, Li X, Li Y, et al. Differentiating T2 hyperintensity in neonatal white matter by two-compartment model of diffusional kurtosis imaging. Sci Rep 2016;6:24473 CrossRef Medline

19. Paydar A, Fieremans E, Nwankwo JI, et al. Diffusional kurtosis imaging of the developing brain. AJNR Am J Neuroradiol 2014;35:808-14 CrossRef Medline

20. Shi J, Chang L, Wang J, et al. Initial application of diffusional kurtosis imaging in evaluating brain development of healthy preterm infants. PLoS One 2016;11:e0154146 CrossRef Medline

21. Shi J, Yang S, Wang J, et al. Detecting normal pediatric brain development with diffusional kurtosis imaging. Eur J Radiol 2019;120:108690 CrossRef Medline

22. Duncan AF, Bann C, Boatman C, et al. NICHD Neonatal Research Network: do currently recommended Bayley-III cutoffs overestimate motor impairment in infants born $<27$ weeks gestation? J Perinatol 2015;35:516-21 CrossRef Medline

23. Vohr BR, Stephens BE, Higgins RD, et al. NICHD Neonatal Research Network: are outcomes of extremely preterm infants improving? Impact of Bayley assessment on outcomes. J Pediatr 2012;161:222-28 CrossRef Medline

24. Bentzley JP, Coker-Bolt P, Moreau NG, et al. Kinematic measurement of 12-week head control correlates with 12-month neurodevelopment in preterm infants. Early Hum Dev 2015;91:159-64 CrossRef

25. Provencher SW. Automatic quantitation of localized in vivo $1 \mathbf{H}$ spectra with LCModel. NMR Biomed 2001;14:260-64 CrossRef Medline

26. Moss HG, Jenkins DD, Yazdani M, et al. Identifying the translational complexity of magnetic resonance spectroscopy in neonates and infants. NMR Biomed 2019;32:e4089 CrossRef Medline

27. Soher BJ, Todd PS, Steinburg J, et al. VeSPA: integrated applications for RF pulse design, spectral simulation and MRS data analysis. In: Proceedings of the Annual Meeting of the International Society for Magnetic Resonance in Medicine, Montreal, Quebec, Canada; May 713, 2011

28. Ades-Aron B, Veraart J, Kochunov P, et al. Evaluation of the accuracy and precision of the diffusion parameter EStImation with Gibbs and NoisE removal pipeline. Neuroimage 2018;183:532-43 CrossRef Medline 
29. Veraart J, Novikov DS, Christiaens D, et al. Denoising of diffusion MRI using random matrix theory. Neuroimage 2016;142:394-406 CrossRef Medline

30. Kellner E, Dhital B, Kiselev VG, et al. Gibbs-ringing artifact removal based on local subvoxel-shifts. Magn Reson Med 2016;76:1574-81 CrossRef Medline

31. Andersson JL, Graham MS, Zsoldos E, et al. Incorporating outlier detection and replacement into a non-parametric framework for movement and distortion correction of diffusion MR images. Neuroimage 2016;141:556-72 CrossRef Medline

32. Andersson JL, Sotiropoulos SN. An integrated approach to correction for off-resonance effects and subject movement in diffusion MR imaging. Neuroimage 2016;125:1063-78 CrossRef Medline

33. Tabesh A, Jensen JH, Ardekani BA, et al. Estimation of tensors and tensor-derived measures in diffusional kurtosis imaging. Magn Reson Med 2011;65:823-36 CrossRef Medline

34. Gudbjartsson H, Patz S. The Rician distribution of noisy MRI data. Magn Reson Med 1995;34:910-14 CrossRef Medline

35. Veraart J, Sijbers J, Sunaert S, et al. Weighted linear least squares estimation of diffusion MRI parameters: strengths, limitations, and pitfalls. Neuroimage 2013;81:335-46 CrossRef Medline

36. Glenn GR, Helpern JA, Tabesh A, et al. Quantitative assessment of diffusional kurtosis anisotropy. NMR Biomed 2015;28:448-59 CrossRef Medline
37. Hansen B, Jespersen SN. Kurtosis fractional anisotropy, its contrast and estimation by proxy. Sci Rep 2016;6:23999 CrossRef Medline

38. Hansen B. An introduction to kurtosis fractional anisotropy. AJNR Am J Neuroradiol 2019;40:1638-41 CrossRef Medline

39. Hui ES, Glenn RG, Helpern JA, et al. Kurtosis analysis of neural diffusion organization. Neuroimage 2015;106:391-403 CrossRef Medline

40. Akaike H. Likelihood of a model and information criteria. Econometrics 1981;16:3-14 CrossRef

41. Burnham KP, Anderson DR. Multimodel inference. Sociological Methods \& Research 2004;33:261-304 CrossRef

42. Urenjak J, Williams SR, Gadian DG, et al. Proton nuclear magnetic resonance spectroscopy unambiguously identifies different neural cell types. J Neurosci 1993;13:981-89 CrossRef Medline

43. Schuff N, Meyerhoff DJ, Mueller S, et al. NAA as a marker of neuronal injury in neurodegenerative disease. Adv Exp Med Biol 2006;576:24162 CrossRef Medline

44. Moffett JR, Arun P, Ariyannur PS, et al. NAA reductions in brain injury: impact on post-injury neuroenergetics, lipid synthesis, and protein acetylation. Front Neuroenergetics 2013;5:11 CrossRef Medline

45. Gadin E, Lobo M, Paul DA, et al. Volumetric MRI and MRS and early motor development of infants born preterm. Pediatr Phys Ther 2012;24:38-44 CrossRef Medline 